\title{
Enfermedad tromboembólica venosa y cirrosis hepática
}

\author{
M. J. García-Fuster, N. Abdilla, M. J. Fabiá, C. Fernández, V. Oliver y M. J. Forner \\ Servicio de Medicina Interna. Hospital Clínico Universitario de Valencia
}

\section{RESUMEN}

Objetivos: la enfermedad tromboembólica venosa (ETV) es poco frecuente en la cirrosis hepática, no estando su tratamiento contemplado en las guías del American College of Chest Physicians. El objetivo del presente trabajo es aportar la experiencia de pacientes cirróticos con ETV hospitalizados en nuestro centro en los últimos 15 años.

Material y método: de enero de 1992 a diciembre de 2007 fueron hospitalizados en nuestro centro 2.074 pacientes con cirrosis hepática. Presentaron una ETV no esplácnica 17, siendo ellos la población a estudio. Se recogen datos epidemiológicos y analíticos: hemograma, VSG, química hemática, coagulación, trombofilia congénita, anticuerpos antifosfolípidos (AAFL) y homocisteinemia. Se valoran factores de riesgo adquiridos, características de la trombosis, el tratamiento y las complicaciones.

Resultados y conclusiones: la ETV no esplácnica se observa en el 0,8\% de pacientes cirróticos. En ellos es frecuente observar hipoalbuminemia, disminución de factores anticoagulantes (ATII, PC, y PS), presencia de AAFL e hiperhomocisteinemia, así como la presencia de factores de riesgo adquiridos: intervenciones quirúrgicas, insuficiencia venosa, inmovilización $e$ infecciones. Tras la anticoagulación, se observan complicaciones hemorrágicas mayores en el $35 \%$ de los pacientes.

Palabras clave: Enfermedad tromboembólica venosa y hepatopatía. Trombosis venosa y cirrosis hepática. Embolia pulmonar y hepatopatía.

\begin{abstract}
Objective: despite the endogenous coagulopathy of cirrhosis, some patients do experience thrombophilic states. The American College of Chest Physicians failed to address the prevention and treatment of venous thromboembolism (VTE) occurring among these patients. This study aims to describe the characteristics of cirrhotics patients hospitalized in the last 15 years, and to use the experience gained.

Material and method: we retrospectively reviewed all admissions for cirrhosis in our hospital from 1992 to 2007. A total of 17 patients had non-portal venous thromboembolic disease. We recorded risk factors, epidemiological and laboratory data, thrombosis characteristics, and treatment complications.

Results and conclusions: approximately $0.8 \%$ of all hospitalized patients with cirrhosis had a non-portal VTE despite the elevated INR and low platelet count. We found low serum albumin, acquired antithrombin III, protein $\mathrm{C}$ and protein $\mathrm{S}$ deficiency, presence of antiphospholipid antibodies, and hyperhomocisteinemia in blood tests. Many patients had hemorragic complications during anticoagulation therapy, and 35\% needed blood transfusions.
\end{abstract}

Key words: Thrombosis and hepatic failure. Coagulopathy and cirrhosis. Venous thromboembolism and hepatopathy.

García-Fuster MJ, Abdilla N, Fabiá MJ, Fernández C, Oliver V, Forner MJ. Enfermedad tromboembólica venosa y cirrosis hepática. Rev Esp Enferm Dig 2008; 100: 259-262.

\section{INTRODUCCIÓN}

La cirrosis hepática se acompaña de anomalías en la hemostasia y coagulación que generalmente dan lugar a

Recibido: 27-08-07.

Aceptado: 12-02-08.

Correspondencia: María José García-Fuster. Servicio de Medicina Interna. Hospital Clínico Universitario de Valencia. Avda. Blasco Ibáñez, 17. 46010 Valencia. e-mail: garciafuster_mjo@gva.es la aparición de fenómenos hemorrágicos, no obstante en ocasiones se crean estados de hipercoagulabilidad con el resultado de trombosis venosa profunda (TVP) y/o embolia pulmonar (EP).

La trombosis de la vena porta, favorecida por mecanismos hemodinámicos de hipertensión en el área esplácnica, es la más frecuente y se observa en el $54 \%$ de las autopsias de estos pacientes (1). En cambio las TVP de las venas no esplácnicas y la EP ocurren en menor cuantía. En la bibliografía encontramos algunas publicaciones de 
casos aislados $(2,3)$ y tan sólo la valoración de la incidencia de la enfermedad, en una serie de pacientes cirróticos con enfermedad tromboembólica venosa (ETV) no esplácnica realizada por Northup y cols. (4) en el año 2006.

La profilaxis y el tratamiento de la ETV en estos pacientes son complicados, debido a los trastornos de la coagulación inherentes a la hepatopatía. Actualmente se abordan de forma empírica, ya que no están incluidos en las guías de actuación del American College of Chest Physicians (5), organismo encargado de elaborar programas de profilaxis y tratamiento de la ETV.

El objetivo del presente trabajo es analizar la incidencia, características clínicas, factores de riesgo, tratamiento y complicaciones de los pacientes cirróticos con ETV hospitalizados en nuestro centro en los últimos 15 años con el fin de aportar experiencias que permitan mejorar el manejo de la enfermedad.

\section{MATERIAL Y MÉTODO}

Se revisó la base informatizada del Hospital Clínico Universitario de Valencia, desde enero de 1992 hasta diciembre de 2007, observando que habían ingresado 2.074 pacientes con el diagnóstico de cirrosis hepática, de ellos 17 presentaban una ETV no esplácnica y son la población a estudio. Los pacientes ingresaron: 7 por la propia ETV, 4 para una intervención quirúrgica, 3 por un cuadro infeccioso y el resto por descompensación de su cirrosis. El diagnóstico de cirrosis hepática se aceptó cuando era secundario a biopsia hepática o se debía a criterios clínicos típicos de la enfermedad: encefalopatía hepática, varices gástricas o esofágicas o ascitis no maligna.

La ETV debía haber ocurrido posterior o simultáneamente al diagnóstico de cirrosis y debía estar documentada por eco-doppler, flebografía, angio-TAC o angio-RNM, en caso de TVP, y por gammagrafía de ventilación-perfusión o/y TAC pulmonar helicoidal cuando se trataba de EP. De las historias clínicas se recogieron antecedentes personales y familiares, datos epidemiológicos y los parámetros analíticos: hemograma, VSG, creatinina, bilirrubina total (BT), transaminasas, gama-glutamil-transpeptidasa (GGT), proteínas totales, albúmina, tiempo de protrombina, I Quick, INR, tiempo de tromboplastina parcial activada (TTPA), tiempo de trombina (TT), fibrinógeno y dímero D (DD), ATIII, proteína C, proteína $\mathrm{S}$, factor V Leiden, mutación de la protrombina G20210A, anticuerpos antifosfolípidos (AAFL): anticardiolipina y/o $\beta_{2}$ glicoproteína y la homocisteinemia. Serología de hepatitis virus B y C. Dado el carácter retrospectivo del estudio, algunos parámetros no estaban valorados en todos los pacientes.

La severidad del daño hepático se evaluó con el índice MELD (5) (Model for End-stage Liver Disease score) según la fórmula: $(11,2 \ln (\mathrm{INR})+3,78 \ln$ (bilirrubina total) $+9,57 \ln$ (creatinina) $+6,43$ y por la clasificación puntuación de Child-Pugh según la encefalopatía, ascitis, bilirrubina $(\mathrm{mg} / \mathrm{dl})$, albúmina $(\mathrm{g} / \mathrm{dl})$ y tasa de protrombina $(\%)$.
En cuanto a las características de la ETV se valoraron: los factores de riesgo adquiridos (inmovilizaciones, intervenciones quirúrgicas, infecciones e insuficiencia venosa), la localización de la trombosis, el tratamiento y las complicaciones.

Se realizó un estudio descriptivo de los valores cuantitativos con medias y DE y los valores cualitativos se expresaron mediante frecuencias.

\section{RESULTADOS}

De los 2.074 pacientes ingresados con cirrosis hepática tuvieron una ETV no esplácnica 17, lo cual supone una incidencia del $0,8 \%$. Eran mujeres 9 de ellos y la edad media de presentación fue de 69 años, rango (4480). Doce tenían serología positiva a hepatitis virus $\mathrm{C} \mathrm{y}$ uno a virus B. En 4 existían antecedentes de alcoholismo crónico. La severidad del daño hepático mostró un índice MELD medio de 12,70 (2,7) y de Child-Pugh de 7,7 $(1,1)$, estadio $B$.

El promedio de los datos analíticos de los 17 pacientes lo podemos ver en la tabla I, donde destaca la plaquetopenia, la hipoalbuminemia, la disminución del INR y el aumento del DD. La valoración de datos de la trombofilia realizada en 5 pacientes la podemos observar en la tabla II, destacando la presencia de AAFL en los 5 pacientes en que se estudió y la disminución de los factores anticoagulantes. Ningún paciente era portador de FV Leiden ni de mutación de

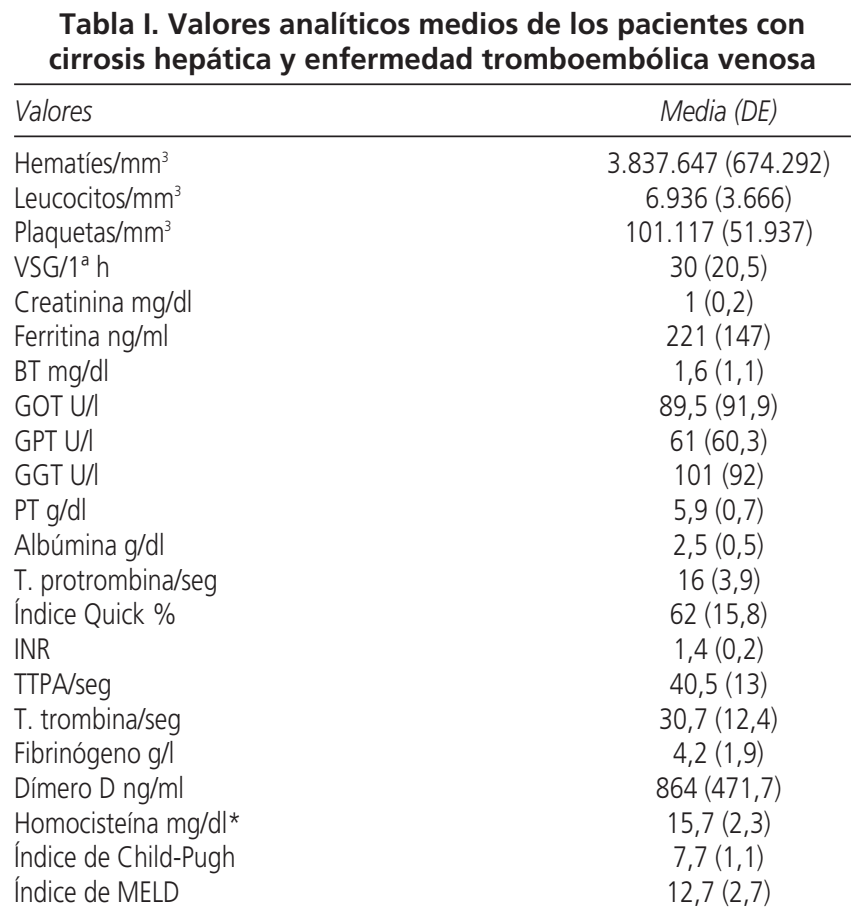

VSG: velocidad de sedimentación globular; BT: bilirrubina total; GOT: glutámico oxalacético transaminasa; GPT: glutámico pirúvico transaminasa; GGT: gamma glutamil transpeptidasa; PT: proteínas totales; INR: cociente internacional normalizado; TTPA: tiempo de tromboplastina parcial activada. *Realizado en 7 pacientes. 
Tabla II. Valores del estudio de trombofilia en 5 pacientes con cirrosis hepática y ETV

\begin{tabular}{lccccc}
\hline Parámetros & $1^{\circ} p$ & $2^{\circ} p$ & $3^{\circ} p$ & $4^{\circ} p$ & $5^{\circ} p$ \\
\hline ATIII & $29 \%$ & $64 \%$ & $46 \%$ & $42 \%$ & \\
Proteína C funcional & $28 \%$ & $60 \%$ & $30 \%$ & $60 \%$ & \\
Proteína S funcional & $46 \%$ & $70 \%$ & $50 \%$ & $65 \%$ & \\
Factor V Leiden & - & - & - & - & \\
Protrombina G20210A & - & - & - & - & + \\
AAFL & + & + & + & + & + \\
\hline
\end{tabular}

ATIII: antitrombina III; AAFL: anticuerpos antifosfolipídicos.

la protrombina. Los datos relativos a la ETV los podemos ver reflejados en la tabla III. Once pacientes presentaron una TVP y siete un EP. En sólo un paciente se presentaron conjuntamente. De las TVP en 7 pacientes se afectaron las venas proximales (poplítea, femoral superficial, común y/o iliacas) y en 4 las venas distales. En 7 ocasiones la trombosis ocurrió en la pierna derecha y en 4 en la izquierda.

Dentro de los factores de riesgo adquiridos, 5 pacientes habían sido intervenidos quirúrgicamente en los 7 días previos, 4 de ellos de cirugía traumatológica y 1 de cirugía abdominal. Un paciente fue inmovilizado tras una fractura de fémur y 7 tenían insuficiencia venosa periférica previa a la trombosis. En 6 pacientes coincidió la ETV con una infección grave, 2 de origen peritoneal y 4 de vías urinarias.

En cuanto al tratamiento, 11 pacientes fueron tratados sólo con heparina de bajo peso molecular (HBPM) y 6 recibieron HBPM durante la primera semana y posteriormente dicumarínicos. Presentaron complicaciones hemo-

Tabla III. Características epidemiológicas, etiopatogénicas y tratamiento de pacientes cirróticos con enfermedad tromboembólica venosa

\begin{tabular}{lc}
\hline Características & $N^{0}$ pacientes \\
\hline ETV & 17 \\
Varones/mujeres & $8 / 9$ \\
TVP & 11 \\
Proximal & 7 \\
Distal & 4 \\
Derecha & 7 \\
Izquierda & 4 \\
EP & 7 \\
Acompañando TVP & 1 \\
Aislado & 6 \\
Factores de riesgo & \\
C. trauma & 4 \\
C. abdominal & 1 \\
Inmovilización & 1 \\
Insuficiencia venosa & 1 \\
Infecciones & 7 \\
Tratamiento & 6 \\
HBPM & \\
HBPM + acenocumarol & 11 \\
Correcto & 6 \\
Complicaciones & 3 \\
Hemorragias mayores & 14 \\
Hemorragias menores & 6 \\
\hline
\end{tabular}

ETV: enfermedad tromboembólica venosa; TVP: trombosis venosa profunda; EP: embolismo pulmonar; HBPM: heparina de bajo peso molecular. rrágicas 14 pacientes $(83 \%)$, siendo graves con necesidad de transfusión en 6 de ellos, el 35\%. Estas hemorragias indujeron a abandonar el tratamiento a la mayoría y sólo 3 pacientes permanecieron anticoagulados durante 6 meses. Una de las hemorragias graves ocurrió durante el tratamiento con HBPM y cinco durante la toma de dicumarínicos. Hubo un caso de recurrencia tras el abandono temprano del tratamiento.

\section{DISCUSIÓN}

En el presente estudio hemos observado que el 0,8\% de los pacientes cirróticos hospitalizados presenta una ETV no esplácnica y que durante el tratamiento anticoagulante el $83 \%$ de los pacientes tiene complicaciones hemorrágicas, siendo en el $35 \%$ de los pacientes de carácter grave. La incidencia de ETV hallada es similar al $0,5 \%$ observado por Northup e inferior al 0,9-4,5\% que presentan los pacientes hospitalizados por otras patologías médicas o al 4-12\% cuando las patologías son quirúrgicas (4).

El sexo no mostró ser un factor selectivo para padecer la enfermedad. Destaca la alta frecuencia en que se observó una EP sin localización de trombosis periférica (el $35 \%$ ) y que la pierna más afecta fue la derecha, localización inhabitual por la disposición anatómica de la arteria sobre la vena iliaca. El índice MELD mostró un valor medio de $12(2,7)$, lo cual supone un pronóstico de supervivencia en los pacientes con ETV superior a los 3 meses (6) y un índice medio de Child-Pugh de 7,7 $(1,1)$ que supone un estadio evolutivo B. Analíticamente, se observa una hipoalbuminemia, hallazgo ya observado por Northup (4) y al que le atribuyó un valor predictivo de ETV en el cirrótico. El estudio de la coagulación mostró un alargamiento del INR, TPTA, TT y una disminución de ATIII, PC, PS, consecuencia de la disfunción del hepatocito que genera menos factores procoagulantes y anticoagulantes. El desequilibrio entre ambos será una de las principales causas de la producción de hemorragias o fenómenos tromboembólicos según el predominio de uno de ellos. La elevación del DD encontrada puede ser secundaria al fenómeno tromboembólico; no obstante es frecuente observar en el paciente cirrótico una elevación del DD por un estado de hiperfibrinólisis secundaria a la disminución de la síntesis del plasminógeno, factor XIII, alfa 2 antiplasmina y TAFI (thrombine activable fibrinolisis inhibitor) y a un menor aclaramiento hepático del t-PA (tissue plasminogen activator) y del PAI (plasminogen activator inhibitor). Este desequilibrio fibrinolítico ha sido considerado como una coagulación intravascular diseminada (CID)like, al no consumir plaquetas ni factor VIII. Últimamente este fenómeno ha sido denominado accelerated intravascular coagulation and fibrinolisis (AICF) (7).

El fibrinógeno mantiene sus cifras normales aunque su función está alterada por unas cadenas $\alpha$ anormales y alto contenido en ácido siálico (8). 
Ningún paciente era portador de FV Leiden ni PTG20210A y la disminución de ATIII, PC y PS se consideró adquirida, dada la ausencia de manifestaciones trombóticas previas a la hepatopatía y la ausencia de enfermedad trombótica familiar.

Es de destacar la presencia de AAFL en los 5 pacientes en que se estudió. El alto poder trombógeno de estos anticuerpos ya es conocido y en estudios previos se había observado que la mitad de los pacientes cirróticos con trombosis portal eran portadores de los mismos (9). Cuatro de los pacientes con AAFL eran virus $\mathrm{C}$ positivos, asociación ya descrita por otros autores. La homocisteinemia presentaba valores superiores a $15 \mathrm{mg} / \mathrm{dl}$ en 5 de los 7 pacientes en que se estudió, siendo un factor trombógeno más a tener en cuenta en la ETV de los cirróticos, hecho también observado por Fernández-Miranda y cols. (10) en los pacientes con enfermedad inflamatoria intestinal.

En cuanto a los factores de riesgo adquiridos transitorios, observamos que en 6 pacientes existían antecedentes de lesiones traumatológicas o intervenciones quirúrgicas, en 7 antecedentes de insuficiencia venosa y en 6 infecciones coincidentes, lo cual implica que el paciente cirrótico está sometido al mismo carácter multifactorial de las trombosis observado por Rossendal y que, por tanto, debe considerarse también su inclusión en las pautas profilácticas.

El tratamiento anticoagulante es complicado, tanto por el desequilibrio de la hemostasia como por los cambios de la farmacodinamia de los anticoagulantes en la insuficiencia hepática. La heparina y los dicumarínicos tienen una vida media alargada por disminución de su metabolización, el déficit de vitamina $\mathrm{K}$ potencia la acción de los dicumarínicos y la disminución de la ATIII da lugar a una disminución de la acción de la heparina. De hecho Carmassi (11) recomienda dar ATIII junto con la heparina para facilitar su acción. Todos estos factores dificultan la anticoagulación de estos pacientes y así en el presente estudio observamos que el $83 \%$ presentó complicaciones hemorrágicas, siendo en el 35\% hemorragias mayores, porcentaje muy por encima del $3 \%$ que se observa en el tratamiento anticoagulante de individuos no hepatópatas. Como consecuencia, 14 de los 17 pacientes no completaron el tratamiento anticoagulante.

El presente trabajo presenta las limitaciones propias de ser retrospectivo y de haber sido realizado con una pe- queña población, consecuencia de la baja incidencia de esta patología, no obstante muestra que la disminución de factores anticoagulantes fisiológicos, la presencia de AAFL, el aumento de la homocisteinemia y la existencia de factores de riesgo adquiridos podrían aumentan el riesgo de sufrir una ETV en los pacientes cirróticos y que el tratamiento anticoagulante presenta un alto porcentaje de complicaciones hemorrágicas. Sería muy conveniente aunar experiencias para crear una guía de actuación profiláctica y de tratamiento para este tipo de pacientes.

\section{BIBLIOGRAFÍA}

1. Oka K, Tanaka K. Intravascular coagulation in autopsy cases with liver diseases. Thromb Haemost 1979; 42: 564-70.

2. Kalambokis G, Svarna E, Tsianos EV. Long-term anticoagulation therapy for a cirrothic patient with recurrent deep venous thrombosis. J Gastroenterol Hepatol 2005; 20: 1803-4.

3. Espiritu J. Pulmonary embolism in a patient with coagulopathy from end-stage liver disease. Chest 2000; 117: 924-5.

4. Northup P, McMahon M, Ruhl A, Altschuler S, Volk-Bednarz A, Caldwell S, et al. Coagulopathy does not fully protect hospitalized cirrhosis patients from peripheral venous thromboembolism. Am J Gastroenterol 2006; 101: 1524-8.

5. Buller HR, Agnelli G, Hull RD, Hyers TM, Prins MH, Raskob GE. Antithrombotic therapy for venous thromboembolic disease. The seventh ACCP conference on antithrombotic and thrombolytic therapy. Chest 2004; 126: 401S-28S.

6. Malinchoc M, Kamath PS, Gordon FD, Peine CJ, Rank J, ter Borg PC. A model to predict poor survival in patients undergoing transjugular intrahepatic portosystemic shunts [see comment]. Hepatology 2000; 31: 864-71.

7. Schrecengost JE, LeGallo RD, Boyd JC, Moons KG, Gonias SL, Rose CE Jr, et al. Comparison of diagnostic accuracies in outpatients and hospitalized patients of D-dimer testing for evaluation of suspected pulmonary embolism. Clin Chem 2003; 49: 1483-90.

8. Caldwell SH, Hoffman M, Lisman T, Macik BG, Northup PG, Reddy $\mathrm{KR}$, et al., and the Coagulation in Liver Disease Group. Coagulation disorders and hemostasis in liver disease: Pathophysiology and critical assessment of current management. Hepatology 2006; 44: 1039-46.

9. Violi F, Ferro D, Basili S, D’Angelo A, Mazzola G, Quintarelli C, et al. Relation between lupus anticoagulant and splachnic venous thrombosis in cirrhosis of liver. BMJ 1994; 309: 239-40.

10. Fernández-Miranda C, Martínez Prieto M, Casis Herce B, Sánchez Gómez F, Gómez González P, Martínez López J, et al. Hiperhomocisteinemia y mutaciones de la metilentetrahidrofolato reductasa $677 \mathrm{C}-\mathrm{T}$ y 1298A-C en pacientes con enfermedad inflamatoria intestinal. Rev Esp Enferm Dig 2005; 97 (7): 497-504.

11. Carmassi F, Morale M, De Negri F, Carrai M. Modulation of hemostatic balance with antithrombin III replacement therapy in a case of liver cirrhosis associated with recurrent venous thrombosis. J Mol Med 1995; 73: 89-93. 